\title{
Pumping Lemmas for Timed Automata
}

\author{
Danièle Beauquier ${ }^{1}$
}

\begin{abstract}
We remark that languages recognized by timed automata in the general case do not satisfy classical Pumping Lemma (PL) well known in the theory of finite automata. In this paper we prove two weaker versions of Pumping Lemma for timed words : a general one (DPL) where iterations preserve the duration of timed word, and another more restricted one, (LPL) when iterations preserve the length of timed word.
\end{abstract}

\section{Introduction}

An automata-theoretic approach to verification of timing requirements of realtime systems has been extensively developped in recent years using timed automata [1], among recent papers we mention [2, 3] which influenced our work. A timed automaton is a finite automaton with a finite set of real valued clocks. The clocks can be reset to zero within the transitions of the automaton and keep track of the time elapsed since the last reset. Some constraints on the clocks are attached both to locations (analogous to states of usual finite automata) and transitions of the automaton.

Timed automata recognize finite or infinite timed words which are right-continuous discrete-valued functions having letters as values. Several papers study timed automata from the perspective of formal languages theory $[1,4]$. Closure properties and some decision problems for deterministic and nondeterministic timed automata have been considered. In [4] a version of Kleene theorem for timed automata has been elaborated. The authors prove that it is necessary to include intersection in the operations which define regular expressions. In this paper we are interested in another classic feature of regular languages, namely in properties of iterations usually called Pumping Lemmas. We prove that the general version of Pumping Lemma does not hold for timed automata, giving a counter-example. This negative result underlines the fact that the introduction of dense time provides to languages recognized by timed automata a more complicated structure. Nevertheless we establish a weak version of Pumping Lemma

${ }^{1}$ Address: University Paris-12, Dept. of Informatics, 61, Av. du Gén. de Gaulle, 94010 Créteil, France.

\#E-mail: beauquier@univ-paris12.fr 
(DPL) where the iteration preserves the duration of the timed word. The part of this result concerning a positive iteration can be found also in [5] (this was pointed out by referees). The dual version (LPL) where the iteration preserves the length of the timed word is proved for a sub-family of timed automata, the strict timed automata.

The paper is organized as follows : in section 2 we recall the definition of timed automata and timed words recognized by timed automata. Section 3 contains a series of lemmas concerning the iteration properties of runs of timed automata which are used in the last section. The last section studies different versions of Pumping Lemma and their status with respect to regular languages.

\section{Timed Automata: Definitions}

\section{$2.1 \quad$ Timed words}

Let $\Sigma$ be a finite alphabet, and $\boldsymbol{R}_{\geq 0}$ be the set of non negative reals. A (finite) timed word is a right-continuous piecewise-constant function $\xi:[0, k) \rightarrow \Sigma$ for some $k \in \boldsymbol{R}_{\geq 0}$ such that $\xi$ has a finite number of discontinuities. If $k$ is equal to $0, \xi$ is the empty word denoted by $\epsilon$. Here we slightly deviate from the definition given in [4], because the right-continuity of timed words seems to better reflect the semantics of the runs of timed automata.

Every timed word $\xi$ can be written (in many ways) $a_{1}^{r_{1}} a_{2}^{r_{2}} \ldots a_{n}^{r_{n}}$, where $a_{i} \in$ $\Sigma, r_{i} \in \boldsymbol{R}_{+}$and $\sum r_{i}=k$ if $\xi(t)=a_{i}$ for $t \in\left[r_{i-1}, r_{i}\right)$. If we impose $a_{i} \neq a_{i+1}$, then the representation of $\xi$ is unique and the length of $\xi$ denoted by $|\xi|$ is equal to $n$ and its duration denoted by $d(\xi)$ is equal to $k$. Length and duration of $\epsilon$ are equal to zero. We denote by $T(\Sigma)$ the set of all finite timed words over the alphabet $\Sigma$.

For every $\xi_{1}, \xi_{2} \in T(\Sigma)$ with respective durations $k_{1}$ and $k_{2}$ their concatenation $\xi_{1} \xi_{2}$ is the timed word $\xi$ with duration $k_{1}+k_{2}$ such that for $t \in\left[0, k_{1}\right), \xi(t)=$ $\xi_{1}(t)$, and for $t \in\left[k_{1}, k_{1}+k_{2}\right) \xi(t)=\xi_{2}\left(t-t_{1}\right)$. Clearly, $\left|\xi_{1} \xi_{2}\right| \leq\left|\xi_{1}\right|+\left|\xi_{2}\right|$ and $d\left(\xi_{1} \xi_{2}\right)=d\left(\xi_{1}\right)+d\left(\xi_{2}\right)$.

For $u=a_{1}^{r_{1}} a_{2}^{r_{2}} \ldots, a_{n}^{r_{n}}$ and a positive integer $p$, denote by $u^{\frac{1}{p}}$ the timed word $a_{1}^{\frac{r_{1}}{p}} a_{2}^{\frac{r_{2}}{p}} \ldots a_{n}^{\frac{r_{n}}{p}}$. We have $\left|u^{\frac{1}{p}}\right|=|u|$ and $d\left(u^{\frac{1}{p}}\right)=\frac{1}{p} d(u)$. Note that $\left(u^{\frac{1}{p}}\right)^{p}=u$ iff $u$ is of the form $a^{r}$ for some $a \in \Sigma$.

A timed language over the alphabet $\Sigma$ is a subset of the set of timed words $T(\Sigma)$.

\section{$2.2 \quad$ Timed automata}

Timed automata were introduced by R. Alur and D. Dill [1].

A timed automaton consists of a finite number of locations supplied with clocks and constraints in terms of equalities and inequalities involving clocks. The edges of the automaton now depend on time, and this makes the automaton more powerful than the classical one.

The clocks of an automaton constitute a finite set of identifiers. Given a set $C$ of clocks, the set of clock constraints, denoted by $\operatorname{guard}(C)$, is the set of formulas of the form: 
- true, false, $c \sim n$ where $c \in C, n \in \boldsymbol{N}$ and $\sim \in\{>,<,=\}$,

- $f_{1} \wedge f_{2}, f_{1} \vee f_{2}$ where $f_{1}$ and $f_{2}$ are formulas in $\operatorname{guard}(C)$.

A timed automaton over $\Sigma$ is a tuple $\mathcal{A}=\left(S, \lambda, \mu, s_{\text {init }}, F, C, E\right)$ where:

- $S$ is a finite set of locations,

- $C$ is a finite set of clocks,

- $\lambda: S \rightarrow \Sigma$ is an output function,

- $\mu: S \rightarrow \operatorname{guard}(C)$, assigns to each location a guard called invariant of the location,

- $s_{\text {init }} \in S$ is the initial location,

- $F \subseteq S$ is a set of final locations,

- $E \subseteq S \times \operatorname{guard}(C) \times 2^{C} \times S$ gives the set of edges between locations labeled by sets of clocks and formulas.

Let $\left(s, s^{\prime}, \phi, \delta\right)$ be an edge from $s$ to $s^{\prime}$. The set $\phi \subset C$ gives the set of clocks to be reset and $\delta$ is a clock constraint in $\operatorname{guard}(C)$ to be satisfied when following this edge.

A clock assignment for a set of clocks $C$ is a function $\nu$ from $C$ to $R$, i. e. $\nu \in \boldsymbol{R}^{C}$. A state of the system is a triple of the form $\langle s, \nu, t\rangle$, where $s \in S, \nu \in \boldsymbol{R}^{C}$, and $t \in \boldsymbol{R}_{\geq 0}$.

By $\nu+t$, where $t \in \boldsymbol{R}$, we denote the clock assignment which assigns to every clock $c$ the value $\nu(c)+t$. In the same way if $A$ is a state $\langle s, \nu, \tau\rangle, A+t$ denotes the state $\langle s, \nu+t, \tau+t\rangle$.

Let $\nu$ be a clock assignment. For $X \subset C$, we denote by $\langle X\rangle \nu$ the clock assignment which assigns 0 to each $c$ in $X$ and agrees with $\nu$ over the rest of the clocks. A transition is a pair of states $\sigma=\left(\langle s, \nu, t\rangle,\left\langle s^{\prime}, \nu^{\prime}, t^{\prime}\right\rangle\right)$ of the automaton $\mathcal{A}$, with an edge $\left(s, s^{\prime}, \phi, \delta\right) \in E$ such that

- $\nu+t^{\prime}-t$ satisfies $\delta$,

- for all $\tau \in\left[0, t^{\prime}-t\right), \nu+\tau$ satisfies $\mu(s)$,

- $\nu^{\prime}=\langle\phi\rangle\left(\nu+t^{\prime}-t\right)$ and $\nu^{\prime}$ satisfies $\mu\left(s^{\prime}\right)$.

The value $t^{\prime}-t$ is called the delay of the transition.

Now let us define a finite run of a timed automaton simply called a run below. A finite run $\rho$ is a pair of sequences $(\mathcal{S}(\rho), \mathcal{E}(\rho))$ such that $\mathcal{S}(\rho)$ is a sequence of states $\left(\left\langle s_{i}, \nu_{i}, t_{i}\right\rangle_{0 \leq i \leq n}\right.$ and $\mathcal{E}(\rho)$ is a sequence of edges $\left(s_{i-1}, s_{i}, a_{i}, \phi_{i}, \delta_{i}\right)_{0<i \leq n}$ satisfying the following requirement:

for every $i=1, \ldots, n$

the pair $\left(\left\langle s_{i-1}, \nu_{i-1}, t_{i-1}\right\rangle,\left\langle s_{i}, \nu_{i}, t_{i}\right\rangle\right)$ with the edge $\left(s_{i-1}, s_{i}, \phi_{i}, \delta_{i}\right)$ is a transition.

The length of the run $\rho$ is equal to $n$ and its duration denoted by $d(\rho)$ is equal to $t_{n}-t_{0}$.

The trace of the run $\rho$ is the timed word $\lambda\left(s_{0}\right)^{t_{1}-t_{0}} \lambda\left(s_{1}\right)^{t_{2}-t_{1}} \ldots, \lambda\left(s_{n-1}\right)^{t_{n}-t_{n-1}}$. Note that the duration of $\rho$ is equal to the duration of its trace but the length of $\rho$ can be treater than the length of its trace.

When the edges are irrelevant, we represent a run as $\left(\left\langle s_{i}, \nu_{i}, t_{i}\right\rangle\right\rangle_{0 \leq i \leq n}$.

A run $\left(\left\langle s_{i}, \nu_{i}, t_{i}\right\rangle\right)_{0 \leq i \leq n}$ is an accepting run if $s_{0}=s_{i n i t}, t_{0}=0, \nu_{0}(c)=0$ for every clock $c$ and $s_{n} \in F$. 
A finite timed word over the alphabet $\Sigma$ is recognized or accepted by the automaton $\mathcal{A}$ if it is the trace of an accepting run of $\mathcal{A}$. The set of finite timed words recognized by the automaton $\mathcal{A}$ is denoted by $L(\mathcal{A})$.

Example. The language accepted by the automaton of Figure 1 where $s_{2}$ is the

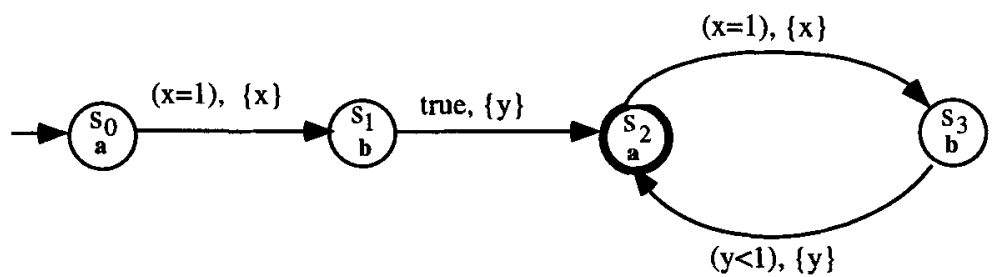

Fig. 1. A timed automaton

final location is:

$$
\begin{gathered}
\left\{a^{r_{0}} b^{q_{0}} a^{r_{1}} b^{q_{1}} \ldots a^{r_{n}} b^{q_{n}} \mid n>0, r_{0}=1, q_{0}<1, \text { for } i=1, \ldots, n q_{i-1}+r_{i}=\right. \\
\left.1 \text { and } r_{i}+q_{i}<1\right\} \cup\left\{a^{r_{0}} b^{q_{0}} \mid r_{0}=1, q_{0}>0\right\} .
\end{gathered}
$$

Here the function $\mu$ is the constant "true". A sample word accepted by the automaton is:

$$
a^{1} b^{0.7} a^{0.3} b^{0.2} a^{0.8} b^{0.1} .
$$

Every timed word accepted by this automaton has the property that the sequence $\left(q_{i}\right)$ of exponents of the letter $b$ is strictly decreasing.

Two finite runs $\rho_{1}$ and $\rho_{2}$ are equivalent if they have the same extremities, i. e. their first and last states are respectively equal: $\mathcal{S}\left(\rho_{1}\right)(0)=\mathcal{S}\left(\rho_{2}\right)(0)$ and $\mathcal{S}\left(\rho_{1}\right)(k)=\mathcal{S}\left(\rho_{2}\right)(l)$ where $k$ and $l$ are the lengths of respectively $\rho_{1}$ and $\rho_{2}$. Clearly, two equivalent runs have the same duration.

\subsection{Clock regions}

The set of states of a timed automaton is infinite. The set of clock regions is a finite set obtained as a quotient of an equivalence relation among the clock assignments. More details about this notion of region is in [1]. Let $K_{0}$ be the greatest constant appearing in the clock constraints of the automaton. Remind that clock constants are natural numbers. For clock assignments $\nu$ and $\nu^{\prime}$ in $\boldsymbol{R}^{C}$ we say that $\nu \equiv \nu^{\prime}$ iff the following conditions are met:

- For each clock $x \in C$ either $\left\lfloor\nu_{x}\right\rfloor$ and $\left\lfloor\nu_{x}^{\prime}\right\rfloor$ are the same, or both are greater than $K_{0}$, 
- For every pair of clocks $x, y \in C$ such that $\nu(x) \leq K_{0}$ and $\nu(y) \leq K_{0}$

1. $f \operatorname{ract}(\nu(x)) \geq \operatorname{fract}(\nu(y))$ iff $\operatorname{fract}\left(\nu^{\prime}(x)\right) \geq \operatorname{fract}\left(\nu^{\prime}(y)\right)$

2. $\operatorname{fract}(\nu(x))=0$ iff $\operatorname{fract}\left(\nu^{\prime}(x)\right)=0$.

The relation $\equiv$ is an equivalence relation, and $[\nu]$ will denote the equivalence class of $\boldsymbol{R}^{C}$ to which $\nu$ belongs. A clock region is such an equivalence class. There are only finitely many such regions. Note that $\nu \equiv \nu^{\prime}$ does not necessarily imply $\nu+t \equiv \nu^{\prime}+t$.

\section{Finite runs of timed automata}

The set of finite runs of a timed automaton has a natural structure of partial monoïd. Given two runs $\rho=\left(\left\langle s_{i}, \nu_{i}, t_{i}\right\rangle_{i=0, \ldots, k}\right)$ and $\rho^{\prime}\left(\left\langle s_{i}^{\prime}, \nu_{i}^{\prime}, t_{i}^{\prime}\right\rangle_{i=0, \ldots, k^{\prime}}\right)$ such that the last state of $\rho,\left\langle s_{k}, \nu_{k}, t_{k}\right\rangle$ is equal to the first state of $\rho^{\prime},\left\langle s_{0}^{\prime}, \nu_{0}^{\prime}, t_{0}^{\prime}\right\rangle$ we define the run $\rho^{\prime \prime}=\rho \rho^{\prime}$ to be as follows:

$\rho^{\prime \prime}=\left(\left\langle s_{i}^{\prime \prime}, \nu_{i}^{\prime \prime}, t_{i}^{\prime \prime}\right\rangle_{i=0, \ldots, k+k^{\prime}}\right)$ where $\left\langle s_{i}^{\prime \prime}, \nu_{i}^{\prime \prime}, t_{i}^{\prime \prime}\right\rangle=\left\langle s_{i}, \nu_{i}, t_{i}\right\rangle$ for $i=0, \ldots, k$ and $\left\langle s_{k+i}^{\prime \prime}, \nu_{k+i}^{\prime \prime}, t_{k+i}^{\prime \prime}\right\rangle=\left\langle s_{i}^{\prime}, \nu_{i}^{\prime}, t_{i}^{\prime}\right\rangle$ for $i=1, \ldots, k^{\prime}$.

Note that it is a partial law. In particular if $\rho$ is a run with positive length, $\rho^{2}$ is never defined because unlike $[1,4]$ we add the absolute time in the states of the timed automaton.

A finite run $\rho=\left(\left\langle s_{i}, \nu_{i}, t_{i}\right\rangle_{i=0, \ldots, k}\right)$ is a pseudo-cycle if $s_{k}=s_{0}$ and $\left[\nu_{k}\right]=\left[\nu_{0}\right]$. The main notion in this part is the notion of conjugation.

Two runs $\rho=\left(\left\langle s_{i}, \nu_{i}, t_{i}\right\rangle_{i=0, \ldots, k}\right)$ and $\rho^{\prime}=\left(\left\langle s_{i}, \nu_{i}^{\prime}, t_{i}^{\prime}\right\rangle_{i=0, \ldots, k}\right)$ of length $k>0$ are conjugate if

$\mathcal{E}(\rho)=\mathcal{E}\left(\rho^{\prime}\right),\left[\nu_{i}\right]=\left[\nu_{i}^{\prime}\right]$ for $i=0, \ldots, k$, and $t_{i}^{\prime}-t_{i-1}^{\prime}=t_{i}-t_{i-1}$ for $i=1, \ldots, k$. Let $n$ be a positive integer and $\rho=\left(\left\langle s_{i}, \nu_{i}, t_{i}\right\rangle_{i=0, \ldots, k}\right)$ and $\rho^{\prime}=\left(\left\langle s_{i}, \nu_{i}^{\prime}, t_{i}^{\prime}\right\rangle_{i=0, \ldots, k}\right)$ be two runs. The run $\rho^{\prime}$ is a $\frac{1}{n}$-conjugate of the run $\rho$ if

$\mathcal{E}(\rho)=\mathcal{E}\left(\rho^{\prime}\right),\left[\nu_{i}\right]=\left[\nu_{i}^{\prime}\right]$ for $i=0, \ldots, k$, and $t_{i}^{\prime}-t_{i-1}^{\prime}=\frac{t_{i}-t_{i-1}}{n}$ for $i=1, \ldots, k$. If $n=1 \rho^{\prime}$ is called simply a conjugate of $\rho$.

From the definition of a $\frac{1}{n}$-conjugate we can deduce:

Lemma 1 (1) If $\bar{\rho}$ is a $\frac{1}{n}$-conjugate of $\rho$, then $d(\rho)=\frac{1}{n} d(\bar{\rho})$.

(2) Given a run $\rho$ with first state $\left\langle s_{0}, \nu_{0}, t_{0}\right\rangle$, and a clock assignment $\nu_{0}^{\prime}$, there is at most one $\frac{1}{n}$-conjugate of $\rho$ with its first state equal to $\left\langle s_{0}, \nu_{0}^{\prime}, t_{0}\right\rangle$.

(3) Two $\frac{1}{n}$-conjugates of a run $\rho$ have the same trace.

Given a finite run $\rho$ there exists at most one run which can be written $\rho_{1} \rho_{2} \ldots \rho_{n}$, where for $i=1, \ldots, n \rho_{i}$ is a $\frac{1}{n}$-conjugate of $\rho$ and $\rho_{1}$ has the same first state as $\rho$. This unique run, when it exists is denoted by $\rho^{[n]}$ and called the $n$-iteration of $\rho$. It satisfies the following property:

Lemma 2 If $\rho^{[n]}$ exists for some $n>1$, then

(1) $d\left(\rho^{[n]}\right)=d(\rho)$ and $\left|\rho^{[n]}\right|=n|\rho|$,

(2) $\rho$ is a pseudo-cycle.

Proof. (1) is clear from the definition of a $1 / n$-conjugate.

To prove (2) note that the last state of $\rho_{1}$ is equal to the first state of $\rho_{2}$, and so it implies that $\rho$ is a pseudo-cycle. 
We say that a clock $c$ crosses an integer value during the run $\rho=\left(\left\langle s_{i}, \nu_{i}, t_{i}\right\rangle\right)_{i \leq n}$ if there is a transition $\left(\left\langle s_{i}, \nu_{i}, t_{i}\right\rangle,\left\langle s_{i+1}, \nu_{i+1}, t_{i+1}\right\rangle\right)$ and some $t \in\left[0, t_{i+1}-t_{i}\right]$ such that $\nu_{i}(c)+t$ is a positive integer.

Clearly we have:

Lemma 3 If a finite run is such that no clock crosses an integer value during $i t$, the value of the delay of any transition of the run is strictly less than 1.

Let $X$ be a subset of clocks. Two states $A=\langle s, \nu, t\rangle$ and $A^{\prime}=\left\langle s, \nu^{\prime}, t^{\prime}\right\rangle$ are $X$-equal if they satisfy :

if $c \in X$ then $\nu(c)=\nu^{\prime}(c)$, and if $c \notin X$ then $\nu(c)$ and $\nu^{\prime}(c)$ are both strictly less than 1 and $\nu(c)=0 \Leftrightarrow \nu^{\prime}(c)=0$.

Transitions where clocks do not cross an integer value have the following basic property:

Lemma 4 Let $\mathcal{A}$ be a timed automaton, $X$ be a subset of clocks, and $A=$ $\langle s, \nu, t\rangle, A^{\prime}=\left\langle s, \nu^{\prime}, t^{\prime}\right\rangle$ be two $X$-equal states of $\mathcal{A}$. Suppose there exists a transition from state $A$ to some state $B$ with edge e and delay $\tau$ where the clocks do not cross an integer value. Suppose at last that for $c \notin X$ we have $\nu^{\prime}(c)+\tau<1$, then there exists a transition from $A^{\prime}$ to some state $B^{\prime}$ with the same edge $e$ and the same delay, moreover $B$ and $B^{\prime}$ are $X$-equal.

Proof. Let us consider the transition from state $A$ to state $B=\left\langle s_{1},\langle\phi\rangle \nu, t+\tau\right\rangle$ with edge $e=\left(s, s_{1}, \phi, \delta\right)$.

For an atomic proposition $c \sim n$ where $\sim \in\{>,<,=\}$ and $n \in N$ consider two cases : $c$ belongs to $X$ or not.

- Case 1: $c \in X$.

For every $\tau^{\prime} \in[0, \tau],\left(\nu^{\prime}+\tau^{\prime}\right)(c)=\left(\nu+\tau^{\prime}\right)(c)$. So $\left(\nu+\tau^{\prime}\right)(c)$ satisfies $c \sim n$ iff $\left(\nu^{\prime}+\tau^{\prime}\right)(c)$ satisfies $c \sim n$.

- Case 2: $c \notin X$.

For every $\tau^{\prime} \in[0, \tau],\left(\nu+\tau^{\prime}\right)(c)$ is strictly less than 1 because in the transition the clocks do not cross an integer value and $\left(\nu^{\prime}+\tau^{\prime}\right)(c)$ is also strictly less than 1. So $\left(\nu^{\prime}+\tau^{\prime}\right)(c)$ satisfies $c \sim n$ iff $\nu+\tau^{\prime}$ satisfies $c \sim n$.

At last, for the same reasons, $\langle\phi\rangle(\nu+\tau)$ and $\langle\phi\rangle\left(\nu^{\prime}+\tau\right)$ satisfy exactly the same set of atomic propositions.

So there is a transition with the edge $e$, the same delay $\tau$, from $A^{\prime}$ to $B^{\prime}=$ $\left\langle s_{1},\langle\phi\rangle\left(\nu^{\prime}+\tau\right), t^{\prime}+\tau\right\rangle$. Clearly, $B$ and $B^{\prime}$ are $X$-equal.

By induction on the length of a run we can deduce the following lemma:

Lemma 5 Let $\mathcal{A}$ be a timed automaton, $X$ be a subset of clocks, and $A=$ $\langle s, \nu, t\rangle, A^{\prime}=\left\langle s, \nu^{\prime}, t^{\prime}\right\rangle$ be two $X$-equal states of $\mathcal{A}$. Suppose there exists a run $\rho$ from $A$ with duration $\tau$ where the clocks do not cross an integer value. Suppose 
at last that for $c \notin X \nu^{\prime}(c)+\tau<1$, then there exists a run $\rho^{\prime}$ conjugate of $\rho$ starting from $A^{\prime}$. Moreover the terminal states of $\rho$ and $\rho^{\prime}$ are $X$-equal.

The reset of a finite run $\left(\left\langle s_{i}, \nu_{i}, t_{i}\right\rangle\right)_{i \leq k}$ is the set of clocks which are reset to 0 during the run, i. e. the union of sets $\phi_{i}$ where $\mathcal{E}(\rho)=\left(s_{i-1}, s_{i}, \phi_{i}, \delta_{i}\right)_{0<i \leq k}$. It is denoted by reset $(\rho)$.

Lemma 6 If a pseudo-cycle $\rho$ is such that no clock crosses an integer value, then $\rho^{[n]}$ exists for every positive integer $n$.

Proof. Let $R$ be equal to $\operatorname{reset}(\rho)$. First we prove that there exists a $\frac{1}{n}$ conjugate $\rho_{1}$ of $\rho$ starting in the same state as $\rho$. Let $\rho=\left(A_{0}, A_{1}, \ldots, A_{p}\right)$, $\mathcal{E}(\rho)=\left(e_{1}, \ldots, e_{p}\right)$ and denote by $t_{i}$ the delay of transition $\left(A_{i-1}, A_{i}\right)$.

We prove that there is a transition from state $A_{0}=\langle s, \nu, t\rangle$ using edge $e_{1}=$ $\left(s, s^{\prime}, \phi_{1}, \delta_{1}\right)$ with delay $t_{1} / n$, to state $A_{1}^{\prime}=\left\langle s^{\prime},\left\langle\phi_{1}\right\rangle\left(\nu+t_{1} / n\right), t+t_{1} / n\right\rangle$.

Let $c>k$ be an atomic proposition and consider a value $\tau \in\left[0, t_{1}\right]$. Then $A_{0}+\tau$ satisfies $c>k$ iff $A_{0}+\tau / n$ satisfies $c>k$, because $c$ cannot cross an integer value during the run. The same for an atomic proposition $c<k$. And a constraint $c=k$ is never satisfied neither by $A_{0}+\tau$ nor by $A_{0}+\tau / n$. At last for the same reason $\left\langle\phi_{1}\right\rangle\left(\nu+t_{1} / n\right)$ and $\left\langle\phi_{1}\right\rangle\left(\nu+t_{1}\right)$ satisfy the same atomic propositions. So there is a transition from $A_{0}$ with delay $t_{1} / n$, using edge $e_{1}$, and arriving in $A_{1}^{\prime}$. Moreover we have $\left[A_{1}^{\prime}\right]=\left[A_{1}\right]$. Indeed, clocks in $\phi_{1}$ are equal to 0 in both $A_{1}$ and $A_{1}^{\prime}$. Let $c, c^{\prime}$ two clocks not in $\phi_{1}$. If $\operatorname{fract}\left(\nu(c)+t_{1}\right) \leq \operatorname{fract}\left(\nu\left(c^{\prime}\right)+t_{1}\right)$ then $\operatorname{fract}\left(\nu(c)+t_{1} / n\right) \leq \operatorname{fract}\left(\nu\left(c^{\prime}\right)+t_{1} / n\right)$ and $\operatorname{fract}(c)$ is non zero in both $A_{1}$ and $A_{1}^{\prime}$. So we have $\left[A_{1}^{\prime}\right]=\left[A_{1}\right]$.

Suppose we have proved there exists a run $\left(A_{0}, A_{1}^{\prime}, \ldots, A_{i}^{\prime}\right)$, with some edges $\left(e_{1}, \ldots, e_{i}\right)$ and delays $t_{1} / n, \ldots, t_{i} / n$ such that $\left[A_{j}^{\prime}\right]=\left[A_{j}\right]$ for $j=1, \ldots, i$. Let $c$ be a clock, and denote by $c_{i}$ its value in $A_{i}$ and by $c_{i}^{\prime}$ its value in $A_{i}^{\prime}$. Let $\tau \in\left[0, t_{i+1}\right]$.

- If $c \notin R$ or if $c$ has not still been reset to zero between $A_{0}$ and $A_{i}$, then $c_{i}=c_{0}+\sum_{j=1, \ldots, i} t_{j}$ and $c_{i}^{\prime}=c_{0}+\sum_{j=1, \ldots, i} t_{j} / n$. States $A_{i}+\tau$ and $A_{i}^{\prime}+\tau / n$ satisfy the same atomic propositions concerning the clock $c$ again because $c$ cannot cross an integer value during the run.

- If $c \in R$ and $c$ has been reset to zero between $A_{0}$ and $A_{i}$ then the values of $c$ in $A_{i}+\tau$ and in $A_{i}^{\prime}+\tau / n$ are strictly less than 1 , thus $A_{i}+\tau$ and $A_{i}^{\prime}+\tau / n$ satisfy the same constraints relative to $c$.

At last, let $\phi_{i+1}$ be the reset of the edge $e_{i+1}$. The same arguments prove that $\left\langle\phi_{i+1}\right\rangle\left(A_{i}+t_{i+1}\right)$ and $\left\langle\phi_{i+1}\right\rangle\left(A_{i}^{\prime}+t_{i+1} / n\right)$ satisfy the same atomic propositions. So there is a transition from $A_{i}^{\prime}$ with edge $e_{i+1}$ and delay $\frac{t_{i+1}}{n}$ and $\left[A_{i}^{\prime}\right]=\left[A_{i}\right]$. We have proved by induction the existence of a $1 / n$-conjugate $\rho_{1}$ of $\rho$ starting in the same state as $\rho$.

Suppose we have defined $\rho_{1}, \ldots, \rho_{i}$ some $1 / n$-conjugates of $\rho$ such that the product $\rho_{1} \ldots, \rho_{i}$ exists, with $i<n$. Let $T$ be the duration of $\rho$. Consider the state $Y_{i}$ which is the last state of $\rho_{i}$. Let $c$ be a clock, and denote by $c_{i}$ its value in $Y_{i}$. If $c \notin R$, then $c_{i}=c_{0}+\frac{i T}{n}$ and if $c \in R$ then its values in $Y_{i}$ and in $A_{0}$ are less than 
1. So we can repeat the same reasoning and prove that there is a $1 / n$-conjugate of $\rho, \rho_{i+1}$ starting in $Y_{i}$. Actually, since $\rho$ is a pseudo-cycle we have $\left[A_{0}\right]=\left[A_{p}\right]$, and on other hand $\left[Y_{i}\right]=\left[A_{p}\right]$ so $\left[Y_{i}\right]=\left[A_{0}\right]$. And the lemma is proved.

Note that in general $\rho^{[n]}$ is not equivalent to $\rho$. We need some synchronization to get two equivalent runs. It is done in the lemma below.

Lemma 7 Let $\rho$ be a run with duration strictly less than 1 such that $\rho=\rho_{1} \beta \rho_{2}$ where $\rho_{1}, \rho_{2}$ are pseudo-cycles with the same reset. Suppose that no clock crosses an integer value during the run $\rho$, then for every positive integer $n$ there exists a conjugate $\overline{\beta \rho_{2}}$ of $\beta \rho_{2}$ such that the run $\rho_{1}^{[n]} \overline{\beta \rho_{2}}$ exists and is equivalent to $\rho$.

Proof. If $n=1$ nothing to prove. Suppose $n>1$.

Let $\rho$ be a run such that $\rho=\rho_{1} \beta \rho_{2}$ where $\rho_{1}$ and $\rho_{2}$ are pseudo-cycles and $\operatorname{reset}\left(\rho_{1}\right)=\operatorname{reset}\left(\rho_{2}\right)=X$. Suppose that $\rho_{1}$ starts in $A$ and finishes in $A^{\prime}$, and $\rho_{2}$ starts in $B$ and finishes in $B^{\prime}$. The run $\rho_{1}$ satisfies the hypothesis of Lemma 6 . So $\rho_{1}^{[n]}$ exists and finishes in some state $Y$. Observe that if $A^{\prime}=\left\langle s_{A^{\prime}}, \nu_{A^{\prime}}, t_{A^{\prime}}\right\rangle$, and $Y=<s_{Y}, \nu_{Y}, t_{Y}>$ then $s_{Y}=s_{A^{\prime}}, t_{Y}=t_{A^{\prime}}$ and states $A^{\prime}$ and $Y$ are $\bar{X}$-equal, where $\bar{X}$ is the complement of $X$. Moreover, we have $\nu_{Y}(c) \leq \nu_{A^{\prime}}(c)$ for every clock $c$.

Denote by $\tau$ the duration of the run $\beta \rho_{2}$. Since $\rho$ has a duration strictly less than 1 we can guarantee that for every clock $c \in X$ we have $\nu_{Y}(c)+\tau \leq \nu_{A^{\prime}}(c)+\tau<1$. On the other hand, the run $\beta \rho_{2}$ is such that no clock crosses an integer value, so applying Lemma 5, we prove that there exists a run $\beta^{\prime} \rho_{2}^{\prime}$, conjugate of $\beta \rho_{2}$, starting in $Y$ and arriving in some state $B^{\prime \prime}$. We claim that $B^{\prime \prime}=B^{\prime}$. Actually the clocks reset during $\rho_{2}$ are also reset during $\rho_{2}^{\prime}$ and have the same value in $B^{\prime}$ and in $B^{\prime \prime}$, because the two runs are conjugate and then the delays are the same. The clocks not reset during $\rho_{2}$ were not reset during $\rho_{1}$ so they have the same value in $Y$ and in $A^{\prime}$ and then also in $B^{\prime}$ and $B^{\prime \prime}$. And we can conclude that $\rho_{1}^{[n]} \overline{\beta \rho_{2}}$ exists and is equivalent to $\rho$.

Lemma 8 Let $\rho$ be a run where no clock crosses an integer value, with duration strictly less than 1 , and such that $\rho=\rho_{1} \beta \rho_{2} x \gamma \rho_{3}$ where $\rho_{1}, \rho_{2}$ and $\rho_{3}$ are pseudo-cycles with the same reset and $|x|=1$. There exists a transition $x^{\prime}$ and a conjugate $\overline{\gamma \rho_{3}}$ of $\gamma \rho_{3}$ such that $\rho_{1} \beta x^{\prime} \overline{\gamma \rho_{3}}$ exists and is equivalent to $\rho$.

Proof. Suppose that $\rho$ contains three disjoint successive pseudo-cycles $\rho_{1}, \rho_{2}, \rho_{3}$ with the same reset. The pseudo-cycle $\rho_{1}$ begins in $A$ and finishes in $A^{\prime}, \rho_{2}$ begins in $B$ and finishes in $B^{\prime}, \rho_{3}$ begins in $C$ and finishes in $C^{\prime}$.

Let $t$ be the duration of $\rho$ from $B$ to $B^{\prime}$ and $B_{1}^{\prime}$ be the successor of $B^{\prime}$ in $\rho$. The transition $\left(B^{\prime}, B_{1}^{\prime}\right)$ has a delay $t_{0}$, and corresponds to some edge $e=\left(s, s_{1}, \phi, \delta\right)$. Let $B=\left(s, \nu_{B}, t_{B}\right), B^{\prime}=\left(s, \nu_{B^{\prime}}, t_{B^{\prime}}\right), B_{1}^{\prime}=\left(s_{1}, \nu_{B_{1}^{\prime}}, t_{B_{1}^{\prime}}\right)$.

Then $t_{B^{\prime}}-t_{B}=t$ and $t_{B_{1}^{\prime}}-t_{B^{\prime}}=t_{0}$.

We will prove that from $B$ there is a possible transition using the edge $e$, with 
a delay equal to $t+t_{0}$, to some state $B_{1}^{\prime \prime}, R$-equal to $B_{1}^{\prime}$, with $R$ being equal to the complement of reset $\left(\rho_{2}\right)$. We have to verify three conditions.

(1) For every $\tau \in\left[0, t+t_{0}\right), \nu_{B}+\tau$ satisfies $\mu\left(s_{B}\right)$

(2) $\nu_{B}+t+t_{0}$ satisfies $\delta$

(3) $\langle\phi\rangle\left(\nu_{B}+t+t_{0}\right)$ satisfies $\mu\left(s_{1}\right)$.

Condition (1) :

Due to the fact that the clocks do not cross an integer value during the run and the run has a duration less than 1 , for every clock $c$ there is a unique interval $[k, k+1)$, with $k \in N$ to which the value of the clock belongs during the whole run $\rho$. Let $c$ be a clock, and $\tau \in\left[0, t+t_{0}\right)$. If $c \in \operatorname{reset}\left(\rho_{2}\right)=\operatorname{reset}\left(\rho_{1}\right)$ then $\left(\nu_{B}+\tau\right)(c)$ is less than 1 as $\nu_{B}(c)$. If $c \notin \operatorname{reset}\left(\rho_{2}\right)$, then $\nu_{B^{\prime}}(c)=\nu_{B}(c)+t$ and $\nu_{B}(c), \nu_{B^{\prime}}(c), \nu_{B^{\prime}}(c)+t_{0}$ belong to the same interval $[k, k+1)$ and $\left(\nu_{B}+\tau\right)(c)$ belongs to this interval. Since $\nu_{B}$ satisfies $\mu\left(s_{B}\right)$ then $\nu_{B}+\tau$ satisfies also $\mu\left(s_{B}\right)$. Condition (2) :

The clocks which do not belong to reset $\left(\rho_{2}\right)$ have the same value in $\nu_{B}+t+t_{0}$ and in $\nu_{B^{\prime}}+t_{0}$. The clocks which belong to reset $\left(\rho_{2}\right)=\operatorname{reset}\left(\rho_{1}\right)$ have a value less than 1 in $\nu_{B}+t+t_{0}$ and in $\nu_{B^{\prime}}+t_{0}$. Since $\nu_{B^{\prime}}+t_{0}$ satisfies $\delta, \nu_{B}+t+t_{0}$ satisfies also $\delta$.

Condition (3) :

In the same way, since $\langle\phi\rangle\left(\nu_{B^{\prime}}+t_{0}\right)$ satisfies $\mu\left(s_{1}\right)$ then $\langle\phi\rangle\left(\nu_{B}+t+t_{0}\right)$ satisfies also $\mu\left(s_{1}\right)$. So there is a transition $x^{\prime}$ from $B=\left\langle s, \nu_{B}, t_{B}\right\rangle$ to $B_{1}^{\prime \prime}=$ $\left\langle s_{1},\langle\phi\rangle\left(\nu_{B}+t+t_{0}\right), t_{B_{1}^{\prime \prime}}\right\rangle$.

And $t_{B_{1}^{\prime \prime}}=t_{B}+t+t_{0}=t_{B}^{\prime}+t_{0}=t_{B_{1}^{\prime}}$.

The main point is that $B_{1}^{\prime}$ and $B_{1}^{\prime \prime}$ are $R$-equal where $R$ is the complement of reset $\left(\rho_{2}\right)$. Indeed

- if $c \in \phi$ then $\nu_{B_{1}^{\prime}}(c)$ and $\nu_{B_{1}^{\prime \prime}}(c)$ are both equal to 0 ,

- if $c \notin \phi$ and $c \notin$ reset $\left(\rho_{2}\right)$ then $\nu_{B_{1}^{\prime}}(c)=\nu_{B^{\prime}}(c)+t_{0}=\nu_{B}(c)+t+t_{0}=\nu_{B_{1}^{\prime \prime}}(c)$, - if $c \notin \phi$ and $c \in \operatorname{reset}\left(\rho_{2}\right)$ then $\nu_{B_{1}^{\prime}}(c)$ and $\nu_{B_{1}^{\prime \prime}}(c)$ are both strictly less than 1 . Let $\tau$ be the duration of $\gamma \rho_{3}$. For every clock $c \in \operatorname{reset}\left(\rho_{1}\right)$, we have $\nu_{B}(c)+t+$ $t_{0}+\tau<1$ since the duration of $\rho$ is strictly less than 1 . So $\nu_{B}(c)+t+t_{0}+\tau=$ $\nu_{B_{1}^{\prime \prime}}(c)+\tau<1$ for $c \in \operatorname{reset}\left(\rho_{1}\right.$, and we can apply Lemma 5 to states $B_{1}^{\prime}$ and $B_{1}^{\prime \prime}$. There is a run $\gamma^{\prime} \rho_{3}^{\prime}$ starting in $B_{1}^{\prime \prime}$, conjugate of $\gamma \rho_{3}$, arriving in some state $C^{\prime \prime}$. Now we prove that $C^{\prime \prime}=C^{\prime}$.

Let $C^{\prime}=\left\langle s_{C^{\prime}}, \nu_{C^{\prime}}, t_{C^{\prime}}\right\rangle$ and $C^{\prime \prime}=\left\langle s_{C^{\prime \prime}}, \nu_{C^{\prime \prime}}, t_{C^{\prime \prime}}\right\rangle$.

Sure, $s_{C^{\prime \prime}}=s_{C^{\prime}}=s$ and $t_{C^{\prime \prime}}=t_{C^{\prime}}$. Compare the values of the clocks in $C^{\prime}$ and $C^{\prime \prime}$, that is $\nu_{C^{\prime}}$ and $\nu_{C^{\prime \prime}}$.

- The clocks which are reset to 0 in $\gamma^{\prime} \rho_{3}^{\prime}$ between $B_{1}^{\prime \prime}$ and $C^{\prime \prime}$ are the same as the clocks reset during the run $\gamma \rho_{3}$ between $B_{1}^{\prime}$ and $C^{\prime}$, and since the durations of the transitions are the same, these clocks have the same value in $C^{\prime}$ and $C^{\prime \prime}$. - The clocks not reset between $B_{1}^{\prime}$ and $C^{\prime}$ in $\gamma \rho_{3}$ (and so between $B_{1}^{\prime \prime}$ and $C^{\prime \prime}$ in $\gamma^{\prime} \rho_{3}^{\prime}$ ) have never been reset between $B$ and $B_{1}^{\prime}$ in $\rho_{2} x$ neither between $B$ and $B_{1}^{\prime \prime}$ in $x^{\prime}$. So their values are the same in $B_{1}^{\prime}$ and $B_{1}^{\prime \prime}$, and then remain the same in $C^{\prime}$ and $C^{\prime \prime}$.

Let $\mathcal{A}$ be a timed automaton, $C$ be its set of clocks, $m$ be the number of regions 
of $\mathcal{A}$ and $K_{0}$ be the constant equal to $\left(22^{|C|}+1\right)(|S| m+1)$.

Proposition 1 If a run $\rho$ with duration strictly less than 1 has a length greater than or equal to $(|C|+1) K_{0}$, then $\rho$ can be written $\alpha \rho_{1} \beta \rho_{2} x \gamma \rho_{3} \eta$ with $|x|=1$ such that:

(1) there exists some transition $x^{\prime}$ and some conjugate $\gamma^{\prime} \rho_{3}^{\prime}$ of $\gamma \rho_{3}$ with the timed word $\alpha \rho_{1} \beta x^{\prime} \gamma^{\prime} \rho_{3}^{\prime} \eta$ equivalent to $\rho$

(2) for every positive integer $n$ there is a conjugate $\overline{x \gamma \rho_{3}}$ of $x \gamma \rho_{3}$ such that $\alpha \rho_{1} \beta \rho_{2}^{[n]} \overline{x \gamma \rho_{3}} \eta$ is equivalent to $\rho$.

Proof. There are at most $|C|$ moments during this run when a clock can cross an integer value because the duration of the run is less than 1 , thus a clock can cross an integer part only once. So since $\rho$ has a length more than $(|C|+1) K_{0}$, there is a finite run $\rho^{\prime}$, part of $\rho$ with length at least $K_{0}=\left(22^{|C|}+1\right)(|S| m+1)$ where no clock crosses an integer value. This run $\rho^{\prime}$ contains $\left(22^{|C|}+1\right)$ disjoint parts which are pseudo-cycles because every run of length $|S| m$ contains a pseudocycle. And among these pseudo-cycles at least three have the same reset. The factor $|S| m+1$ in $K_{0}$ instead of $|S| m$ ensures that the pseudo-cycles are not only disjoint but are separated by at least one transition, what justifies the existence of transition $x$. Then we apply Lemmas 7 and 8 .

\section{Pumping Lemmas}

Here we discuss some versions of "Pumping Lemma" for a given language $L \subset$ $S(\Sigma)$, as a natural extension of the classical one [6]. There are two versions according to considering "large words" with respect to their duration or to their length. For timed words the classical Pumping Lemma could be stated as follows: Pumping Lemma Property (PL) There exists a constant $K>0$ such that for every timed word $u \in L$ with length (respectively duration) more than $K$, there exist timed words $v, w, z, w \neq \epsilon$ which satisfy:

$$
u=v w z \text { and for every integer } n \geq 0, v w^{n} z \in L .
$$

Proposition 2 There is a timed automaton $\mathcal{A}$ such that $L(\mathcal{A})$ does not satisfy (PL).

Proof. Consider the automaton of Figure 1. Suppose it satisfies Pumping Lemma Property (PL) for the "duration" version. Let $K$ be a constant for which the property holds. There exists a timed word $u$ in $L(\mathcal{A})$ with $d(u)>K$. We can choose $u$ of the form $a^{r_{0}} b^{q_{0}} a^{r_{1}} b^{q_{1}} \ldots, a^{r_{p}} b^{q_{p}}$ with $p>0$. By our assumption there are words $v, w, z$ such that $u=v w z$ and $v w^{n} z \in L$ for every integer $n \geq 0$. Several cases are to be considered for $w$.

If $w=a^{r}, r>0$, there exists an integer $n$ such that $n r>1$. So $u w^{n} z$ does not belong to $L(\mathcal{A})$. The same if $w=b^{r}, r>0$. 
If $w$ contains both letters $a$ and $b$, then $w^{2}$ cannot be a factor of a word in $L(\mathcal{A})$ because the sequence of exponents of the letter $b$ is not decreasing. Thus $v w^{2} z \notin L(\mathcal{A})$. And $L(\mathcal{A})$ does not satisfy (PL) for the "duration" version.

A similar reasoning can be done if $|u|>K$. Thus $L(\mathcal{A})$ does not satisfy the Pumping Lemma Property (PL) for the "length" version.

In [4] it is proved that the family of languages recognized by timed automata satisfies some Kleene property. In their regular expressions the authors include the intersection. It is not surprising, because a classical Kleene theorem without intersection in regular expressions would imply easily the Pumping Lemma (PL) for languages recognized by timed automata.

Nevertheless using properties established in section 3 we can elaborate some weak versions which will hold. There is a first version when an iteration can be done increasing the duration of the timed word but conserving its length. We prove that for a sub-family of timed automata, the strict automata, some Pumping Lemma holds.

A timed automaton on an alphabet $\Sigma$ is strict if two adjacent locations have different labellings by $\lambda$. Strict timed automata are less expressive as it is proved by the following example. The timed language recognized by the automaton of Figure 2 is

$$
\left\{a^{r} \mid r \in(2,+\infty) \cup\{1\}-\{3\}\right\}
$$

If a strict automaton recognizes such a language it has a single location and it is easy to prove that no such a strict automaton exists.

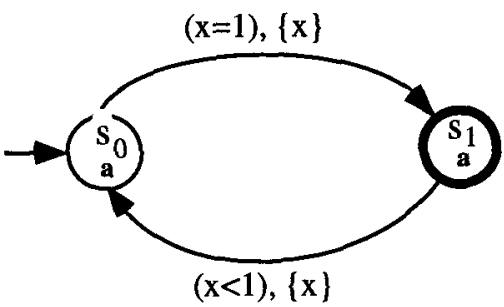

Fig. 2. A counter-example

Lemma 9 If a timed word $u$ is accepted by a strict timed automaton then the lengths of the runs which accept $u$ are equal and equal to the length of $u$.

Proof. Due to the fact that the automaton is strict, the length of a run is exactly the length of the word it recognizes. 
We give first a Pumping Lemma (LPL) which holds for languages recognized by strict timed automata. In this version, the iteration increases the duration of the word but conserves its length.

Proposition 3 Pumping Lemma (LPL) Let L be a language recognized by a strict timed automaton $\mathcal{A}$. There exists a constant $K>0$ such that for every timed word $u \in L$ with duration $d(u)>K|u|$, there exist $v, w \in T(\Sigma), a \in \Sigma$, $r>K$ such that $u=v a^{r} w$ with $r>K$ and $v a^{r^{\prime}} w \in L$ for every $r^{\prime}>K$.

Proof. Denote by $K$ the greatest integer appearing in the guards and the invariants of the automaton $\mathcal{A}$. Let $u$ be a word recognized by $\mathcal{A}$ such that $d(u)>K|u|, u$ can be written $a_{1}^{r_{1}} a_{2}^{r_{2}} \ldots, a_{p}^{r_{p}}$, where $a_{i} \in \Sigma, r_{i} \in \boldsymbol{R}_{+}, a_{i} \neq$ $a_{i+1}$, and every run which accepts $u$ has length equal to $p$. There exists some $i \in\{1, \ldots, p\}$ such that $r_{i}>K$. Let $\rho$ be an accepting run which recognizes the word $u$, it can be written as $\rho_{1} x \rho_{2}$ where $x$ is some transition with delay $r_{i}$ from a state $A=\langle s, \nu, t\rangle$ to some state $\left\langle s^{\prime}, \nu^{\prime}, t+r_{i}\right\rangle$, with an edge $e=\left(s, s^{\prime}, \phi, \delta\right)$ and $\mu(s)=a_{i}$. The main point is that $K$ is the greatest integer appearing in the guards and the invariants of the automaton $\mathcal{A}$. For this reason there is a possible transition $x^{\prime}$ from $A$ using the edge $e$ and with a delay $r^{\prime}$ for every $r^{\prime}>K$. Let $B^{\prime}$ be the end of this transition. Every clock $c$ has either a value zero in both $B$ and $B^{\prime}$ (if $c \in \delta$ ), or a value greater than $K$ in both $B$ and $B^{\prime}$. Due to this fact, there exists a conjugate $\rho_{2}^{\prime}$ of $\rho_{2}$ starting in $B^{\prime}$ and since $\rho_{1} x \rho_{2}$ was an accepting run, $\rho_{1} x^{\prime} \rho_{2}^{\prime}$ is also an accepting run, and thus $v a^{r^{\prime}} w \in L$ for every $r^{\prime}>K$.

One can formulate another version of Pumping Lemma, when an iteration can

be done in a way that increases (in general) the length of the timed word but conserves its duration.

Proposition 4 Pumping Lemma (DPL) Let $L$ be a language recognizable by a timed automaton $\mathcal{A}$. There exists a constant $K>0$ such that the following properties hold. For every word $u \in L$ with length greater than $(\lfloor d(u)\rfloor+1) K$ there exist $v_{1}, v_{2}, v_{3} \in T(\Sigma), v_{2} \neq \epsilon, a \in \Sigma$ and a real $r>0$ such that:

(1) $u=v_{1} v_{2} a^{r} v_{3}$

(2) $v_{1}\left(v_{2}^{\frac{1}{n}}\right)^{n} a^{r} v_{3} \in L$ for every positive integer $n$

(3) $v_{1} a^{r+d\left(v_{2}\right)} v_{3} \in L$.

Proof. Let $K=(|C|+1) K_{0}$ be the constant of Proposition 1. Consider a run $\rho$ of the automaton $\mathcal{A}$ which accepts $u$. The length of $\rho$ is at least $(\lfloor d(u)\rfloor+1) K$, and its duration is $d(u)$. Thus, there is a finite part $\rho^{\prime}$ of $\rho$ of length $K$ and with duration strictly less than 1 . Then we apply to this run $\rho^{\prime}$ Proposition 1. The run $\rho^{\prime}$ can be written $\alpha \rho_{1} \beta \rho_{2} x \gamma \rho_{3} \eta$. So $\rho$ is some $\alpha^{\prime} \alpha \rho_{1} \beta \rho_{2} x \gamma \rho_{3} \eta \eta^{\prime}$. And $\rho$ recognizes a timed word $v_{1} v_{2} a^{r} v_{3}$ where :

$-v_{1}$ is the trace of $\alpha^{\prime} \alpha \rho_{1} \beta$

$-v_{2}$ is the trace of $\rho_{2}$

$-a^{r}$ is the trace of $x$ 
$-v_{3}$ is the trace of $\gamma \rho_{3} \eta \eta^{\prime}$.

Now, there is some $\alpha \rho_{1} \beta \rho_{2} x^{\prime} \gamma^{\prime} \rho_{3}^{\prime} \eta$ which is equivalent to $\rho^{\prime}$ so $\alpha^{\prime} \alpha \rho_{1} \beta \rho_{2} x^{\prime} \gamma^{\prime} \rho_{3}^{\prime} \eta \eta^{\prime}$ is equivalent to $\rho$ and recognizes the word $v_{1} a^{r+d\left(\rho_{2}\right)} v_{3}$. Recall that the delay of transition $x^{\prime}$ is equal to the delay of $x$ plus the duration of $\rho_{2}$. So $v_{1} a^{r+d\left(v_{2}\right)} v_{3} \in L$.

In the same way, there is a conjugate $\overline{x \gamma \rho_{3}}$ of $x \gamma \rho_{3}$ such that for every positive integer $n \alpha \rho_{1} \beta \rho_{2}^{[n]} \overline{x \gamma \rho_{3}} \eta$ is equivalent to $\rho^{\prime}$. Therefore $\alpha^{\prime} \alpha \rho_{1} \beta \rho_{2}^{[n]} \overline{x \gamma \rho_{3}} \eta \eta^{\prime}$ is equivalent to $\rho$ and recognizes $v_{1}\left(v_{2}^{\frac{1}{n}}\right)^{n} a^{r} v_{3}$, and $v_{1}\left(v_{2}^{\frac{1}{n}}\right)^{n} a^{r} v_{3} \in L$.

\section{Remarks}

In part (3) of Proposition 4 we cannot claim that $v_{1} a^{r} v_{3}$ belongs to $L(\mathcal{A})$. That is we cannot suppress directly the factor $v_{2}$, we have to increase at the same time the exponent of $a$.

Part (2) of Proposition 4 claims that if a timed word $u$ is large enough compared to its duration, then some factor $v_{2}$ of $u$ can be replaced by $\left(v_{2}^{\frac{1}{n}}\right)^{n}$.

\section{Example}

We give here an example of application of this Pumping Lemma, to prove that some language cannot be recognized by a timed automaton.

Consider the timed language

$$
L=\left\{a^{r_{0}} b^{r_{1}} a^{r_{2}} b^{r_{3}} \ldots a^{r_{2 n}} b^{r_{2 n+1}} \mid r_{0}>r_{1}>\ldots>r_{2 n+1}, n \geq 0\right\}
$$

Suppose that $L$ is recognized by some timed automaton, and let $K$ be the constant of Proposition 4 for this timed automaton.

There exist in $L$ words with an arbitrarily great length and simultaneously an arbitrarily small duration. So there is a timed word $u$ in $L$ such that $|u|>$ $K(\lfloor d(u)\rfloor+1)$. By Proposition 4, $u$ can be written $u=v_{1} v_{2} x^{r} v_{3}, x \in\{a, b\}$, $v_{2} \neq \epsilon$, and $v_{1} x^{r+d\left(v_{2}\right)} v_{3} \in L, v_{1}\left(v_{2}^{\frac{1}{n}}\right)^{n} x^{r} v_{3} \in L$. But clearly $v_{1} x^{r+d\left(v_{2}\right)} v_{3} \notin L$ neither $v_{1}\left(v_{2}^{\frac{1}{n}}\right)^{n} x^{r} v_{3}$ if $n \neq 1$. Therefore $L$ cannot be recognized by a timed automaton. Note that this property cannot be used considering the untimed language associated to $L$ which is $(a b)^{+}$and so is a regular language.

\section{Conclusion}

We have proved that languages recognized by timed automata do not satisfy classical Pumping Lemma Property, but only weaker versions. This result can be used to prove that a language is not recognizable by a timed automaton by proving that the language does not satisfy this weak Pumping Lemma. We have used also this result to prove that model-checking is decidable for a class of problems formulated in a rather high-level language [7].

Acknowledgments I thank the anonymous referees for their numerous and sound remarks. 


\section{References}

1. R. Alur and D. Dill. A theory of timed automata. Theoretical Computer Science, 126:183-235, 1994.

2. A. Henzinger and O. Kupferman. From quantity to quality. In Hybrid and RealTime Systems Proceeding of HART'97, pages 48-62. Springer Verlag, 1997. Lect. Notes in Comput. Sci., vol. 1201.

3. F. Wang. Parametric timing analysis for real-time systems. Information and Computation, 130:131-150, 1996.

4. E. Asarin, P. Caspi, and O. Maler. A kleene theorem for timed automata. In IEEE Computer Society, LICS'97, pages 160-171, 1997.

5. T. Wilke. Automaten und Logiken fur zeitabhangige Systeme. Ph.d. thesis, Kiel University, 1994.

6. J. E. Hopcroft and J.D. Ullman. Introduction to Automata Theory, Languages and Computation. Addison-Wesley, 1979.

7. D. Beauquier and A. Slissenko. Decidable model checking for a class of timed automata specified in first order logic. Technical Report 97-11, University Paris-12, Department of Informatics, 1997. 